\title{
Erosion de cavitation : méthodes pratiques de prédiction au stade du projet
}

\author{
Cavitation erosion : practical methods for forecasting damage \\ at the design stage \\ par Yves Lecoffre \\ YLec Consultants (La Tronche)
}

Jean-Pierre Franc

LEGI/IMG (Saint Martin d'Hères)

Cavitation erosion is the final consequence of a number of highly complex physical phenomena. The geometry of the hydraulic equipment, its operating conditions including the flow velocity and cavitation $\sigma$ as well as the nature and history of the fluid are the key parameters which govern the size and type of vapor cavities. These cavities are entrained by the flow and collapse against the neighboring solid boundaries. When strong enough, each of these collapses may create a small pit in the material. This pit formation is called the incubation period. The cumulative effect of these mumerous impacts is responsible of fatigue at the surface of materials and this finally causes material removal.

It is practically imposssible to simulate all these phenomena by available computer models or correlations. The only possible way to forecast cavitation damage is to use reduced scale models and apply proven scaling laws.

The most complex part of the scaling concerns the hydrodynamic phenomena. It has been shown that they were satisfactorily represented on models provided the scaling parameters are within a reasonable range and the test fluid is correctly prepared. This may imply nuclei injection.

This hypothesis has been used to derive scaling laws on the flow geometry, the rate of occurence of vapor cavities and their capability to produce pits on solid materials during the so-called incubation period. The histogram of the pits produced can be a measure of the damage capability of the flow. Finally, it has been shown experimentally that this histogram of pits, or at least part of it could be transposed to other operating conditions of a similar flow.

\section{IINTRODUCTION}

L'érosion de cavitation est un phénomène redouté par les fabricants et les exploitants de composants hydrauliques, en raison du manque de méthodes fiables et prouvées de prédiction des dégâts. Les fabricants de machines tournantes cherchent à éviter autant que possible le phénomène en améliorant les tracés et la réalisation des appareils. L'apport des méthodes de calcul a été de ce point de vue fondamental. Il n'en reste pas moins que de nombreux problèmes subsistent, ne serait-ce qu'en raison de la grande variabilité des conditions de fonctionnement.

L'érosion de cavitation est toujours la limite au dimensionnement des machines de transformation d'énergie, au contraire des hélices dont on cherche à limiter le bruit et les vibrations qu'elles engendrent. L'érosion apparaît en effet bien avant les chutes de performances [1]. Une machine hydraulique bien dessinée, outre le fait que ses performances doivent être optimales, sera donc une machine pouvant pré- senter de la cavitation, mais dont l'érosion sera maîtrisée. Plus on sera proche des conditions critiques et plus la machine sera compacte. Elle sera donc, a priori, moins onéreuse.

Les fabricants disposent d'un arsenal de méthodes pour dimensionner les machines :

- les calculs d'écoulements ont fait des progrès rapides et notables. Ils sont souvent néanmoins limités à la détermination des vitesses et pressions moyennes en écoulement monophasique. Le problème de la cavitation, même stationnaire, n'est que rarement considéré.

- la banque de données s'enrichit naturellement par l'accroissement du nombre de machines en service. C'est évidemment un outil précieux pour effectuer des corrélations. L'utilisation des cartes donnant les types de cavitation en fonction des paramètres réduits est une méthode de dimensionnement courante.

- les essais de composants en modèle réduit ou non permettent de caractériser de manière fiable les propriétés dyna- 
miques des écoulements cavitants, comme les conditions d'apparition, les chutes de performances ou même le bruit. Les méthodes de mesure et de transposition deviennent de plus en plus raffinées. On prend par exemple en compte les effets des germes de cavitation en injectant des microbulles dans certains essais standard [2].

- les matériaux ont également fait des progrès considérables et de nombreuses recherches sont en cours sur ce sujet. On cherche à améliorer leur résistance à la cavitation et à la corrosion. De nouveaux revêtements plastiques ou métalliques ont été récemment développés.

Il semble néanmoins que les seules méthodes actuellement utilisées par les constructeurs font appel à des corrélations par rapport à des expériences similaires. Le fabricant d'un composant ne connaît donc pas la marge dont il dispose visà-vis d'une éventuelle érosion. L'incertitude est de plusieurs ordres de grandeur en terme de durée de vie. Des dégâts peuvent apparaître sur des périodes allant de l'heure à quelques années, voire ne jamais apparaitre. Les incertitudes sur la durée de vie s'étendent donc typiquement sur 5 décades.

On a longtemps pensé qu'en raison de la complexité des phénomènes, les corrélations étaient les seules méthodes utilisables. On cherchait donc à limiter le développement de la cavitation ou les vitesses d'écoulement de manière à maintenir le phénomène dans des limites jugées acceptables. Une fois la machine mise en service, on ne pouvait qu'attendre et constater la présence ou non d'érosion. Les résultats des nombreuses études relatives à ce phénomène sont pratiquement peu utilisées par les industriels. Il est vrai qu'elles sont souvent partielles et à caractère parfois académique. Néanmoins, devant les sommes considérables engagées en France dans ces recherches pendant ces vingt dernières années, l'utilisateur contribuable peut avoir des raisons de s'interroger sur leurs retombées pratiques. Cela est d'autant plus vrai que la plupart des thèmes d'étude sont relatifs à l'élaboration de " méthodes de prédiction de l'érosion de cavitation".

Nous tenterons dans ce court article de faire le point sur les dites méthodes en analysant le problème qu'elles abordent et qu'elles tentent de résoudre. A l'heure actuelle, aucune de ces procédures ne donne une réponse totalement satisfaisante à la prédiction de la durée de vie d'une machine, soit parce qu'elles sont trop partielles, soit parce que des chaînons manquent pour finaliser une procédure.

\section{II — LES MÉCANISMES CONDUISANT À L'ÉROSION DE CAVITATION - APPROCHE ANALYTIQUE.}

\section{- 2.1. Les étapes du processus de destruction.}

Nous ne donnerons pas ici le détail des études réalisées sur ce sujet. Nous renvoyons le lecteur aux ouvrages en référence 1 et 3 qui traitent du problème et des paramètres qui l'influencent.

Le mécanisme d'érosion par cavitation passe par les étapes schématisées sur la figure 1. [4]

\subsection{Dimensionnement du composant et conditions de fonctionnement.}

Les étapes 1 et 2 donnent les conditions aux limites du phénomène. On peut éventuellement agir sur elles en modi- fiant les machines, en les installant de manières différentes, en modifiant les points de fonctionnement ou en couplant différemment les composants d'un circuit. Nous ne détaillerons pas les techniques à mettre en œuvre qui font plus partie de la conception hydraulique des machines et de l'ingénierie des installations que de l'analyse du phénomène d'érosion. Notons simplement que l'informatisation des méthodes dédiées à ces problèmes a fait de grands progrès et permet de mettre en œuvre des procédures d'optimisation très sophistiquées.

2.3. Développement de la cavitation, formation et collapse des cavités

Le composant et ses conditions de fonctionnement étant choisis, on doit donc accepter la présence de cavitation et chercher à en prévoir les dégâts potentiels.

On doit, dans un premier temps, détecter la présence ou non de cavitation et en caractériser la localisation, le type et le développement. On sait en effet que certains types de cavitation sont plus agressifs que d'autres et que, selon les conditions de pression qu'elles rencontrent lors de leur collapse, les cavités de vapeur sont ou non susceptibles de créer des dégâts. [1]

\subsubsection{Méthodes numériques.}

Les méthodes numériques ont surtout été développées pour réaliser des calculs en écoulement subcavitant et souvent près du point de meilleur rendement. On citera par exemple Dugué [2] qui précise, dans le cas des hélices marines, qu' " en projet, aucun outil de modélisation de cavitation n'est actuellement utilisé pour la conception ".

Les méthodes numériques utilisées par les concepteurs de pompes permettent de calculer les écoulements visqueux subcavitants stationnaires dans toute la gamme de fonctionnement des machines, même hors adaptation. C'est par exemple le cas du code N3S d'EDF.

Le calcul des poches de vapeur en géométrie 3D se fait actuellement en fluide parfait avec le code MOCA3D du LEGI issu du code CALECHE développé par Métraflu, I'EDF et la SEP. On peut même calculer ces poches en fluide visqueux, à l'aide du code URANUS de l'EPFL.

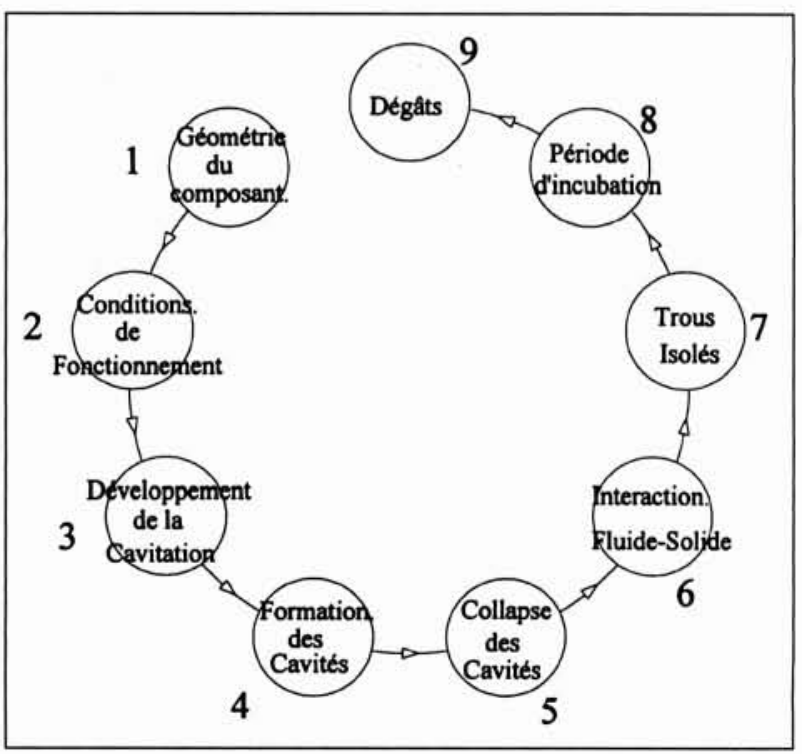

1. Les 9 étapes conduisant à l'érosion. 


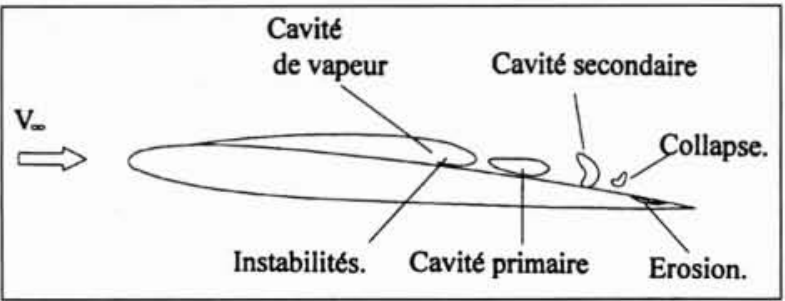

2. Hydrodynamique de l'érosion, cavitation à poches.

Ces codes de calcul sont actuellement limités aux écoulements stationnaires, mais ils peuvent fournir des conditions aux limites intéressantes pour l'analyse de l'entraînement de la vapeur et des collapses associés, en particulier l'épaisseur maximale des poches et le gradient de pression moyen dans la zone de fermeture.

Parallèlement à ces codes à finalité industrielle, les laboratoires cités ci-dessus développent des modèles destinés à caractériser l'entraînement de vapeur à l'aval des cavités [5]. Leur application principale visée est celle du fonctionnement des pompes cryogéniques, mais certains aspects sont directement utilisables pour caractériser l'érosion par cavitation.

Pour la cavitation à poches, malgré ces progrès spectaculaires, on est donc encore très loin de modéliser totalement la cavitation instationnaire, passage obligé vers la prédiction des dégâts.

En cavitation à bulles, par contre, dont on a montré qu'elle était particulièrement agressive en raison de la taille importante des cavités de vapeur, il existe depuis une vingtaine d'années des modèles simples basés sur l'équation de Rayleigh-Plesset [6]. Ils prennent en compte les interactions des bulles entre elles et permettent de caractériser l'évolution de leur dimension en fonction du temps. Ce sont ces modèles qui ont permis de vérifier les lois en $\lambda^{3}$ sur les populations de germes de cavitation [1]. On peut aussi réaliser des estimations des chutes de performances en utilisant ce modèle. On peut enfin déterminer des vitesses caractéristiques des parois de ces bulles en fin de collapse, ce paramètre étant un bon indicateur de l'intensité érosive d'une cavité.

\subsubsection{Les essais.}

La seule méthode actuellement fiable pour prévoir le développement de la cavitation et la plupart de ses effets, consiste encore à utiliser des essais à échelle réduite ou non.

Pour les machines de grandes dimensions, telles les turbines ou les hélices de navires, le recours à l'échelle réduite est nécessaire. Les machines plus petites, telles certaines pompes ou composants passifs, peuvent être testés à l'échelle 1 .

Les essais en modèle réduit ont connu en France un développement spectaculaire, grâce à la mise en œuvre de nouveaux moyens d'essais ou à l'amélioration d'installations existantes. Cela s'est accompagné de techniques nouvelles de métrologie faisant de plus en plus appel à l'informatique.

Parmi les techniques nouvelles utilisées en standard sur le tunnel GTH du bassin des carènes, qui constitue l'investissement français le plus important dans ce domaine, on peut citer le contrôle d'air libre et dissous [2], les mesures de vitesse par anémométrie laser, la prise de vue stéréoscopique des cavités [7] et les mesures acoustiques diverses. On a même pensé développer des méthodes d'essais entièrement automatiques capables de donner, outre les performances des hélices, toutes les indications nécessaires à la caractérisation de la géométrie stationnaire et instationnaire de la cavitation.

On admet en général que, moyennant un certain nombre de précautions, la géométrie des écoulements sur modèle réduit est homothétique à celle du réel, ce qui permet de prévoir ses performances dynamiques stationnaires (les performances) et instationnaires (les vibrations et le bruit). Il en va de même pour la prédiction de l'érosion, puisque les dégâts sont la conséquence ultime de phénomènes d'impacts répétés. On doit considérer que le modèle réduit contient toute l'information nécessaire à la caractérisation de l'agressivité de l'écoulement au réel.

Il reste probablement un champ à explorer qui consisterait à utiliser les techniques de caractérisation hydrodynamique de la cavitation actuellement mis en cuvre pour les adapter à la prédiction de l'érosion. Le bruit, trop souvent présenté comme une méthode absolue, est un indicateur intéressant de ce point de vue [8].

On donne en particulier sur la figure 3 une comparaison entre la densité de trous comptés par méthode optique de diamètre supérieur à un certain seuil et le nombre d'impacts dont la force est supérieure à une certaine valeur. La corrélation est évidemment excellente.

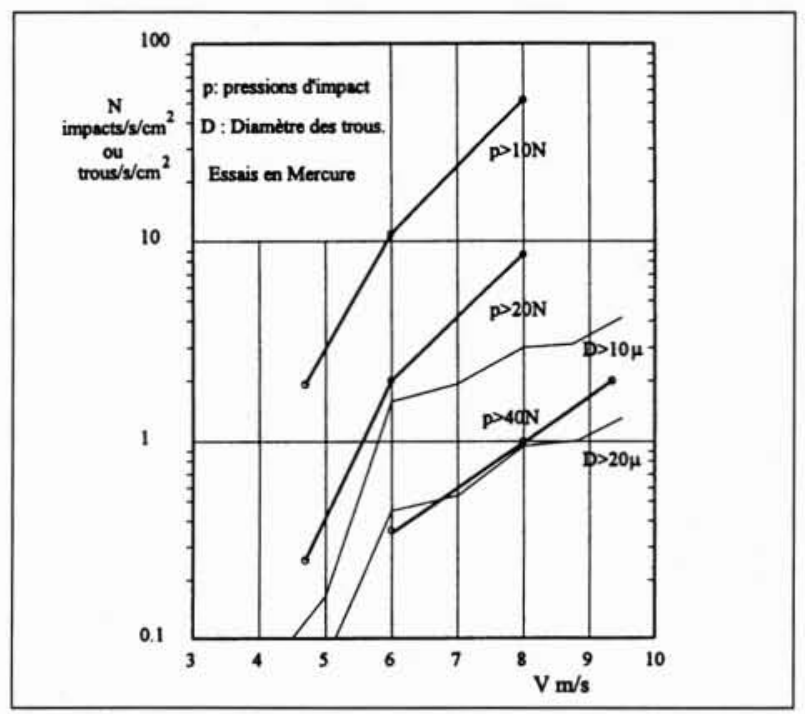

3. Comparaison entre les impulses de pression et les trous. Cas du mercure.

\subsection{Du collapse à la formation des trous.}

Aussi curieux que cela puisse paraitre, on ne connaît pas encore avec certitude le mécanisme responsable de la création de trous dans le matériau, que ce soit du point de vue du fluide ou du matériau. La raison en est peut-être que les impacts de cavitation dans un écoulement se produisent très rapidement, la durée typique de l'onde acoustique créée par un impact étant de 1 microseconde ou moins. Le caractère aléatoire de leur distribution spatio-temporelle fait qu'il est pratiquement impossible de les localiser dans un écoulement et d'instrumenter la surface sur laquelle ils vont se produire.

Pour ces raisons ont été développés plusieurs équipements qui permettent de créer des impacts relativement reproductibles. Citons la Veine Tourbillon Neyrtec (Actuellement appelée Caversim) [9], le Cavermod du LEGI [10] ou les générateurs de bulles de l'Université de Marseille [11]. Ce 
dernier type d'équipement a déjà été utilisé par nombre d'expérimentateurs, à la suite de Lauterborn [12]. Selon les équipements utilisés, ou leur mode de mise en œuvre, on constate des comportements très divers, une cavité de vapeur étant susceptible de produire un ou plusieurs impacts de dimensions très variées.

La plupart des auteurs s'accordent à conclure au peu d'efficacité des microjets qui se produisent en fin de collapse, certains attribuant les dégâts au second rebond des bulles, d'autres au seul effet du premier collapse.

Il est, en tout cas admis par la plupart des auteurs que les effets résultent de phénomènes d'ondes de choc. On a pu en effet montrer que, lorsqu'on utilisait des fluides différents, eau, mercure ou sodium, les vitesses d'apparition des premiers trous étaient proportionnelles à l'impédance acoustique du fluide. Par exemple, pour l'écoulement considéré, les premiers impacts obtenus en eau se produisaient à $12 \mathrm{~m} / \mathrm{s}$ sur de l'acier $316 \mathrm{~L}$ et à seulement $1 \mathrm{~m} / \mathrm{s}$ avec du mercure. Des visualisations d'ondes de choc ont pu être réalisées aussi bien dans la veine tourbillon [13] que dans le cas de collapses de bulles produites par laser.

Les modes de déformation du matériau sous l'effet d'impulsions de pression ont été également étudiés de manière très détaillée au moyen de modèles de calcul faisant interagir une onde de pression dans le liquide et le matériau [14]. On en déduit la déformation plastique du matériau. Bien entendu, ce modèle est approximatif et le restera tant que les mécanismes détaillés de l'interaction fluide-matériau ne seront pas décrits, mais il permet de caractériser l'impact en tenant compte des effets instationnaires d'ondes dans les deux milieux. Sous réserve de comparaison avec les expériences disponibles, on peut espérer caractériser la résistance des matériaux sous impacts et peut-être prévoir les histogrammes de trous créés par la cavitation lorsqu'on change de vitesse et de matériau. On aurait alors un moyen simple d'interpréter les résultats obtenus en modèle réduit et de les transposer au réel, avec d'autres vitesses, d'autres dimensions et d'autres matériaux.

Notons que d'autres modélisations concernant l'interaction fluide-matériau ont été proposées, dont celle qui consiste à expliquer les déformations obtenues, malgré les faibles vitesses des parois des bulles au collapse par des mécanismes de focalisation d'ondes dans le matériau [15].

\subsection{La période d'incubation}

Le mécanisme d'érosion se caractérise par l'apparition de trous individualisés de très faibles dimensions (Pitting) dès que le matériau est soumis à l'écoulement. Après quoi, sous l'effet cumulé des impacts, les trous formés se chevauchent, puis le matériau se dégrade par fatigue et on constate un arrachement de matière.

On a, depuis longtemps [16] [17] [18] considéré que cette période d'incubation était intéressante pour caractériser le phénomène et on a pu montrer que l'évolution des densités d'impacts ou du volume déformé (MDDR) suivaient des lois en puissance de la vitesse. L'originalité de la démarche NEYRTEC [1] a été de faire l'hypothèse que ces grandeurs caractéristiques pouvaient se transposer d'un écoulement à un autre à condition que ceux-ci soient géométriquement semblables.

Dans un premier temps, on a pu montrer que la densité des impacts se transposait selon des lois simples lorsqu'on changeait la vitesse de l'écoulement, le matériau, le fluide et la dimension. Par la suite, en utilisant des observations semiquantitatives, on a pu établir la similitude des histogrammes de trous obtenus pour un même matériau à la même vitesse d'écoulement, mais avec une échelle différente.

Ces règles de similitude, dites en $\left(\lambda, \lambda^{3}\right)$ sont à la base de la méthode globale qui sera exposée ci-dessous.

Parallèlement à ces résultats scientifiques ont été développées des méthodes diverses de préparation d'échantillons par polissage ou dépôts divers. Ont également et surtout été mis en œuvre des systèmes d'analyses adaptés au problème. Le premier d'entre eux est la méthode optique par interférométrie sous microscope du LOBE qui a une définition verticale de l'ordre de $0,01 \mu \mathrm{m}$.

On peut donc dire que les recherches françaises ont permis de développer les règles de similitude relatives à la période d'incubation, ainsi que les techniques expérimentales permettant d'en mesurer les paramètres caractéristiques. II s'agit de la première méthode quantitative applicable à l'étude de l'érosion de cavitation.

\subsection{Les dégâts}

Après cette période d'incubation, on constate un arrachement de matière qui constitue l'érosion proprement dite. Là encore, des études ont été réalisées dans le but de comprendre les mécanismes mis en jeu.

L'apport le plus important est sans aucun doute dû à Karimi de l'EPFL [19], qui a montré que le mécanisme d'arrachement de matière provenait d'un durcissement superficiel résultant d'un écrouissage sous l'action répétée des impacts. Un modèle de comportement tenant compte des propriétés physiques des matériaux a été proposé et les essais malheureusement limités destinés à le valider se sont montrés très encourageants.

D'un point de vue expérimental, les résultats récents obtenus au CETIM [20] dans le cadre d'une étude réalisée pour I'AFCP montrent que les taux d'arrachement de matière sont assez proches des vitesses de déformation (MDDR) obtenues pendant la période d'incubation. L'essai a été réalisé sur la veine standard NEYRTEC. Cela a été obtenu pour 3 matériaux, Cupro Aluminium, Acier $316 \mathrm{~L}$ et acier $13 / 4$, le coefficient de proportionnalité entre ces deux vitesses caractéristiques de la dégradation des matériaux étant compris entre 1 et 5 et dépendant peu de la vitesse pour un matériau donné. Bien qu'il faille être prudent quant aux conclusions à donner à de tels essais, en particulier si on change de dimension, il est intéressant de noter que la vitesse de déformation est, dans ces cas, du même ordre de grandeur que la vitesse d'arrachement. C'est en tout cas la première fois que de tels essais de longue durée sont réalisés et où ces deux valeurs intégrales peuvent être comparées. L'une des raisons est qu'une telle série d'essais nécessite des milliers d'heures de fonctionnement à grande vitesse.

\begin{tabular}{|l|c|c|c|}
\hline MDDR/MDPR & $316 \mathrm{~L}$ & $13 / 4$ & Cupro-Alu \\
\hline $63 \mathrm{~m} / \mathrm{s}$ & 4 & 2,2 & \\
\hline $50 \mathrm{~m} / \mathrm{s}$ & 4,9 & 1,91 & \\
\hline $40 \mathrm{~m} / \mathrm{s}$ & & 1,15 & 1,93 \\
\hline
\end{tabular}

4. Rapport des vitesses de déformation (MDDR) du matériau aux vitesses d'arrachement (MDPR). 


\section{III — LES MÉTHODES GLOBALES}

\subsection{Les principes}

Les méthodes globales consistent à modéliser en tout ou partie les 9 étapes du processus d'érosion par cavitation. Certains raccourcis sont parfois utilisés et permettent d'éviter une analyse détaillée des phénomènes. L'objectif est, dans tous les cas, de définir l'agressivité de l'écoulement, souvent caractérisée par un endommagement du matériau.

\subsection{Les approches théoriques globales.}

Il s'agit de mettre en œuvre des modèles décrivant l'ensemble du processus de dégradation.

La première tentative en ce sens a été faite par Kato et al [20] qui a posé les bases d'une telle méthode en en précisant les limites. Il utilise seulement 6 étapes au lieu des 9 que nous proposons, mais il considère que l'étape $\mathrm{N}^{\circ} 1$ est l'estimation de la longueur de cavité. La différence essentielle est que ces auteurs ne passent pas par l'intermédiaire de la période d'incubation. Ils considèrent que l'histogramme des impacts caractérisés par des forces impulsionnelles est suffisant pour en déduire l'érosion.

Nous avons nous-mêmes appliqué une démarche similaire dans le cas de cavitation de vannes, dans le but d'évaluer les risques d'érosion qu'elles présentent [21]. Les vannes sont assimilées à des diaphragmes et on peut caractériser l'écoulement à partir des paramètres de cavitation et de la vitesse d'écoulement. En se donnant un point de fonctionnement jugé, a priori critique, et doté d'une échelle de risque égale à $1\left(\sigma_{1}=0,5, \mathrm{~V}=30 \mathrm{~m} / \mathrm{s}\right.$ pour l'acier inoxydable $\left.316 \mathrm{~L}\right)$, on calcule les risques associés à d'autres conditions de fonctionnement. Cette procédure est applicable à la maintenance d'un parc de vannes, par exemple dans l'industrie nucléaire.

\subsection{La similitude}

En 1980, NEYRTEC a proposé d'utiliser des relations de similitude pour transposer les dégâts subis par les matériaux du modèle au prototype. Il s'est avéré que ce modèle, malgré sa simplicité, permet de réaliser des transpositions d'histogrammes de trous de cavitation obtenus pendant la période d'incubation à la condition que les écoulements soient homothétiques, que les vitesses d'écoulement sur modèle et au réel soient identiques et que les matériaux, au moins pour les alliages métalliques les plus couramment utilisés le soient également. Ces lois de transposition sont dites en $\left(\lambda, \lambda^{3}\right)$ et sont décrites en [1].

L'objectif initial était de " donner aux industriels des moyens de prédire l'érosion à terme à partir d'essais de courte durée en modèle réduit ${ }^{\prime \prime}$.

\subsection{Les principes}

La plupart des machines et des composants hydrauliques font l'objet de tests en modèle réduit ou non dans les conditions de cavitation proches de celles du fonctionnement réel. On admet sans trop de difficultés que les écoulements sur modèle sont suffisamment représentatifs pour qu'on puisse extrapoler les performances aux conditions du prototype, au moins au premier ordre, c'est-à-dire lorsque les paramètres de cavitation, $\sigma$, sont identiques. Les techniques expérimentales les plus récentes prennent en compte des phénomènes de second ordre, comme la teneur en germes de l'eau ou la structure des couches limites.
Lorsqu'on réalise des études acoustiques, on admet implicitement que ces extrapolations valent aussi bien pour les aspects instationnaires que stationnaires. Cela suppose simplement que les géométries spatiales et temporelles des écoulements, incluant le comportement instationnaire des plus petites cavités de vapeur sont identiques sur modèle et au réel.

Si on considère que l'érosion de cavitation résulte du collapse de cavités de vapeur, qui répondent aux lois de similitude des écoulements fluides, on peut dire que l'écoulement modèle contient toute l'information nécessaire à la prédiction de l'érosion au réel.

Il était donc légitime de chercher à caractériser par un moyen adapté l'agressivité hydrodynamique de l'écoulement modèle et à extrapoler les résultats obtenus aux conditions réelles. L'analyse détaillée de la similitude montre que l'on peut réaliser tout ou partie de cette extrapolation dans le cas de deux écoulements géométriquement semblables lorsque l'on change de dimension, de vitesse, de matériau ou de fluides.

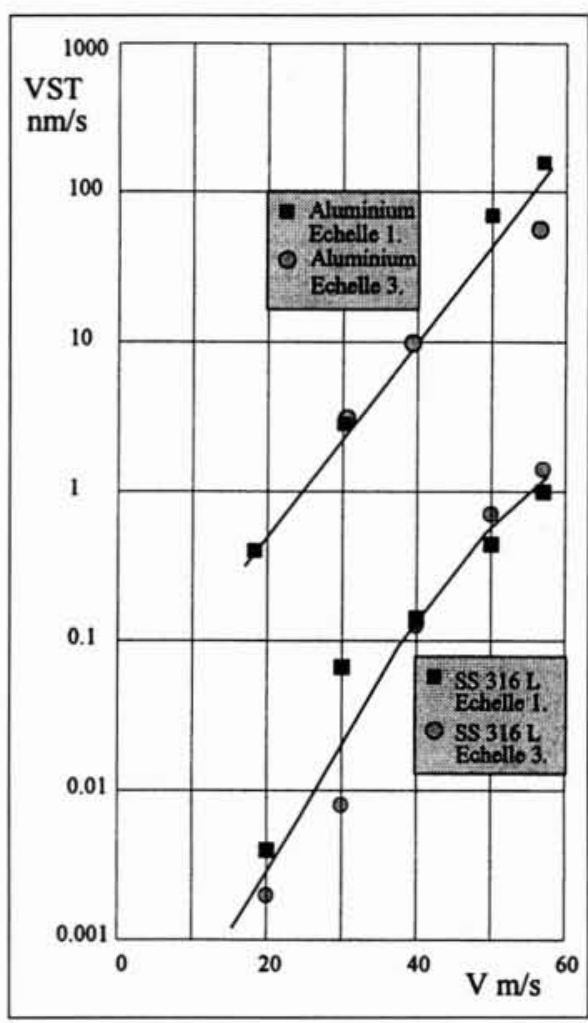

5. Vitesse moyenne VST de pénétration des impacts en fonction de la vitesse d'écoulement et de la taille des échantillons. Essais en eau sur veine standard.

Dans le cas particulier d'écoulements à même vitesse, avec un même fluide sur un même matériau, à des échelles différentes, on a pu montrer [4] que les histogrammes d'impacts obtenus pendant la période dite d'incubation étaient directement transposables par application des lois de similitude en $\left(\lambda, \lambda^{3}\right)$. Les figures 5 et 6 donnent les résultats récemment obtenus par ACB dans le cas de l'Aluminium et de l'acier inoxydable dans le cadre du programme Européen BriteEuram. La vitesse de déformation VST (ou MDDR) pendant la période d'incubation ne dépend que du matériau et de la vitesse. La dimension des impacts est, quant à elle, simplement proportionnelle au rapport des échelles.

1. Y.LECOFFRE, Proposition technique à la DRET. Mai 1980. 


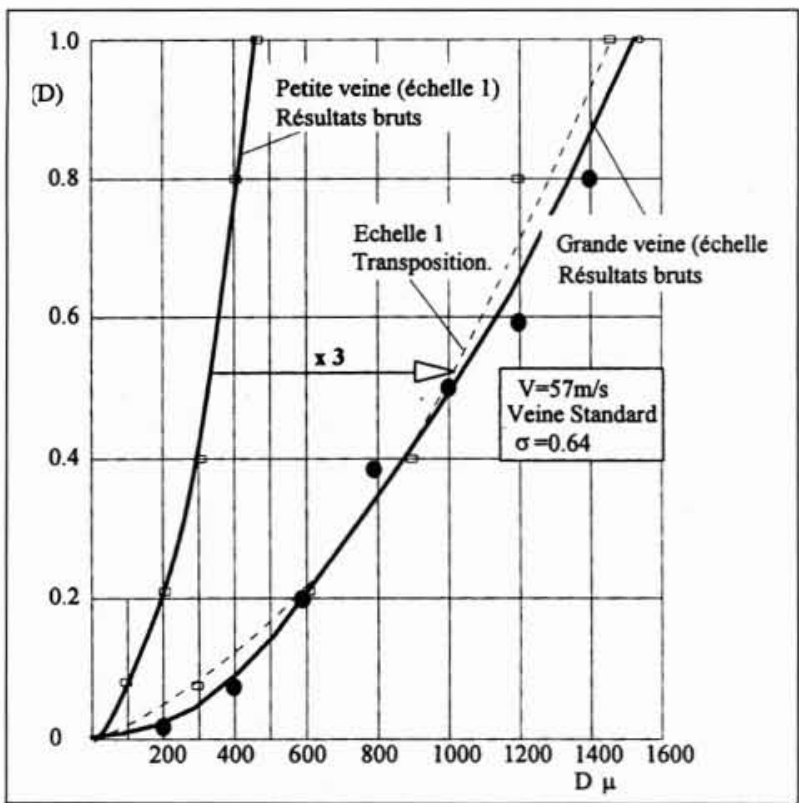

6. Contribution de chaque classe de diamètre à la déformation des échantillons pendant la période d'incubation. Transposition en fonction des dimensions.

Ces résultats correspondent exactement aux modèles théoriques, et ceci malgré l'incroyable complexité des phénomènes intermédiaires. Ils prouvent que I'histogramme d'impacts obtenu pendant la période d'incubation peut être utilisé comme une mesure de l'agressivité érosive et que cet histogramme est simplement transposable moyennant quelques précautions.

On dispose ainsi de données expérimentales qui permettent de réaliser des extrapolations sans avoir à passer par le détail des étapes d'analyses intermédiaires développées cidessus. Dans le processus général décrit sur la figure 1, on effectue un saut de l'étape 2 à l'étape 7.

Actuellement, la validité de la méthode n'est prouvée que pour un même matériau, avec un même fluide et des vitesses égales, le paramètre de similitude étant la dimension. II serait, à notre avis, assez facile de l'étendre au cas le plus couramment rencontré dans les laboratoires industriels, à savoir des essais à échelle et à vitesse réduites. Il serait alors utile de mettre en œuvre un matériau de test moins résistant qu'au réel et développer une procédure adaptée de transposition.

La durée typique d'un tel test serait de l'ordre de quelques minutes à une heure. Ceci est donc tout à fait envisageable dans une procédure d'essais standard.

\subsubsection{Prévision des dégâts.}

Nous rappelons sur la figure 7 la méthode globale de prédiction des dégâts que nous avions proposée. Elle consiste à transposer aux conditions réelles les résultats du test en modèle réduit. L'histogramme de trous ainsi obtenus doit alors être reproduit au moyen d'une méthode accélérée sur le matériau réel.

L'une des techniques de simulation accélérée peut consister à utiliser un écoulement proche du réel, produisant un histogramme analogue au précédent, mais il s'agit d'une technique lourde et onéreuse qui requiert des temps d'essais très importants.

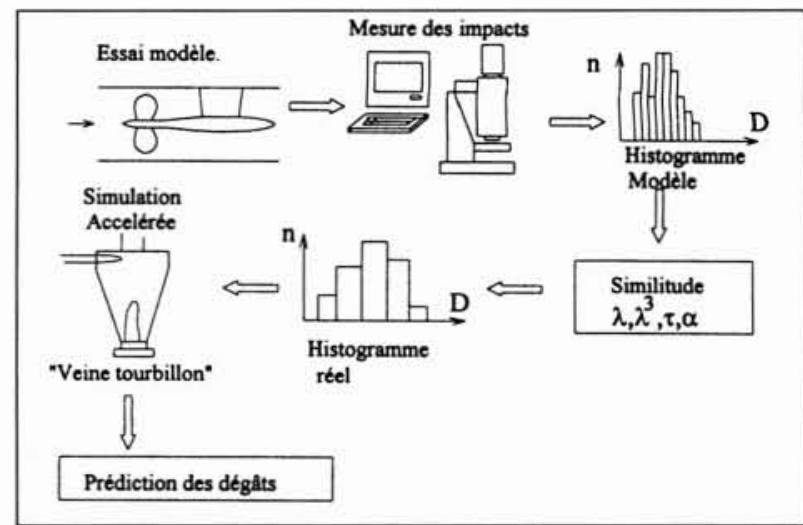

7. Schéma logique d'une méthode de prédiction de l'érosion par cavitation.

On peut aussi utiliser les résultats obtenus par le CETIM, qui consistent à supposer que l'érosion est maximalisée si on prend en compte la vitesse de déformation comme vitesse d'érosion. Cette approche devrait être validée par des essais complémentaires prenant en compte les dimensions générales des impacts, mais elle constitue actuellement un pis aller pour certains matériaux.

On peut enfin, comme nous l'avions suggéré dans la méthode générale, développer un appareil de simulation d'histogrammes performant. Le principe pourrait en être la veine tourbillon [9], mais celle-ci devrait être notablement améliorée pour que l'on puisse créer des impacts calibrés à la demande en faisant simplement varier les conditions aux limites de l'écoulement, fréquence des impacts, pressions et débit. Un tel développement est envisageable et l'appareil pourrait, soit être une veine tourbillon améliorée, soit être un montage de table dont les conditions aux limites seraient parfaitement contrôlables (Simuler).

\section{IV $\square$ CONCLUSION}

Les développements effectués en France dans le domaine de l'érosion de cavitation ont été nombreux. Ils ont montré que, malgré l'extrême complexité des phénomènes impliqués dans le processus, il était possible d'envisager une méthode de prédiction utilisable par les bureaux d'études et les laboratoires industriels dès la phase de conception des composants.

Les méthodes de prédiction purement théoriques peuvent être utilisées si on cherche simplement à évaluer les risques et à optimiser la dimension des composants. Leur niveau de prédiction n'est pas encore suffisant, car elles impliquent une connaissance parfaite de l'ensemble des 9 étapes du processus.

La plupart des méthodes pratiques permettent de court-circuiter certaines des étapes. C'est par exemple le cas des méthodes acoustiques par mesures d'impacts qui sont très prometteuses. Elles permettent de passer de l'étape 2 à 5 , car elles caractérisent le collapse des cavités. A notre avis, l'objectif serait de pouvoir relier les signaux acoustiques et les histogrammes de trous. On aurait ainsi un accès immédiat à la phase 7, qui est en fait la caractérisation de l'histogramme d'intensités hydrodynamiques.

Actuellement, la seule méthode globale permettant de caractériser l'érosion de manière quantitative est la mesure des histogrammes de trous. Des techniques de mesure spécifiques ont été développées en France qui montrent la faisabi- 
lité de cette procédure et des règles de transposition ont été démontrées. Pratiquement, on peut prévoir l'érosion à terme à partir d'essais de courte durée, ce qui était l'objectif initial de ces recherches. Il reste à définir une méthode expérimentale plus renseignée permettant de réaliser l'extrapolation des histogrammes lorsque les vitesses du modèle sont plus faibles qu'au réel. De plus, un simulateur d'érosion plus performant que la veine tourbillon devrait être développé.

On voit donc que l'on dispose, grâce aux efforts continus de la communauté scientifique française, et à des programmes de recherche menés souvent en partenariat avec des équipes étrangères, d'un arsenal de moyens complémentaires qui permettrait de résoudre ce vieux problème de prédiction de l'érosion, longtemps considéré comme trop complexe pour pouvoir être abordé dans les processus de dimensionnement. Quelques efforts et collaborations supplémentaires permettraient de les marier pour créer un outil répondant exactement aux souhaits et aux budgets des laboratoires industriels.

Souhaitons pour le XXIe siècle que les nouvelles méthodes de financement de la recherche technologique française et européenne permettent encore de compléter de tels programmes à long terme et d'en initier de nouveaux.

\section{REMERCIEMENTS}

Les auteurs remercient la DRET, l'EDF, la Commission Européenne et les divers participants à ce programme qui a débuté en 1980.

\section{RÉFÉRENCES}

[1]. Yves LECOFFRE. La Cavitation, traqueurs de bulles. Editions HERMES, Paris. 1994.

[2]. Christian DUGUE, Problèmes de cavitation dans l'industrie Navale. Hydrodynamique des procédés industriels diphasiques, Colloque d'hydrotechnique, SHF, Toulouse, 28 et 29 Janvier 1998.

[3]. J.P. FRANC et al : 1995. La Cavitation, Mécanismes physiques et aspects industriels. Presses Universitaires de Grenoble.

[4]. Yves LECOFFRE. Cavitation Erosion, Hydrodynamic Scaling Laws. Practical Method of Long Term Damage Prediction. International Symposium on Cavitation, Deauville 1995.

[5]. M.CALLENAERE, J.P.FRANC and J.M.MICHEL : Influence of cavity thickness and pressure gradient on the unsteady behaviour of partial cavities. Third Int Symp on Cavitation. Grenoble, France, 1998.

[6]. Yves LECOFFRE, Cavitation, Bubble Trackers, Balkema Publishers, Rotterdam. 1998.

[7]. V.GODEFROY, D.FRECHOU. Digital Image Processing For Cavitation On Marine Propellers. Third Int Symp on Cavitation. Grenoble, France, 1998.

[8]. J.P.FRANC and J.M.MICHEL . Cavitation Erosion Resaerch In France : The State Of The Art. Franco-Japanese Meeting On Cavitation Erosion, Sendai, December 1996.

[9]. Yves LECOFFRE. Erosion de cavitation, vers une méthode de prédiction. Revue scientifique et technique de la défense, ler trimestre 1992.

[10]. El-Galhia FILALI, J.M.MICHEL, S. FUJIKAWA, S. HATORI. The modified Cavermod Device : Hydrodynamics, Force Measurements and Erosion Tests. Third Int Symp on Cavitation. Grenoble. France. 1998.

[11]. J.C. ISSELIN. A.P.ALLONCLE, M.AUTRIC. Investigations of Material Damage Induced by an Isolated Vapor Bubble. Third Int Symp on Cavitation. Grenoble, France, 1998.

[12]. C.D. OHL, O.LINDAU, W.LAUTERBORN, A.PHILIPP. Details of asymmetric bubble collapse. Third Int Symp on Cavitation. Grenoble. France, 1998.

[13]. AVELLAN F., FARHAT M., Dynamique des cavités érosives : étude du collapse des vortex cavitants. SHF 1988.

[14]. FORTES PATELLA R and REBOUD J.L. A New Approach to Evaluate the Cavitation Erosion Power. To be published in J.F.M.

[15]. Y. LECOFFRE et B. VALIBOUSE. Erosion par Cavitation et Focalisation des ondes de Surface. Analogie avec les Surfaces Libres. La Houille Blanche 1994

[16] KNAPP R.T., "Recent Investigations of Cavitation and Cavitation Damage", Transactions of ASME, Vol. 77, 1955, p. 1045-1054.

[17]. STINEBRING DR, Arndt R E A and Holl, J.W. Scaling laws of cavitation damage. Journal of hydronautics, Vol 11, 1977.

[18]. KATO H., "A Consideration on Scaling Laws of Cavitation Erosion", International Shipbuilding Progress, Vol. 22, 1975, p. 305-327.

[19]. KARIMI A. " Modèle mathématique pour la prédiction de la vitesse d'érosion." La Houille blanche 7/8 1988.

[20]. B. Le FUR and J.F. DAVID. Comparison Between Pitting Rate and Erosion Rate for 3 Materials. Third Int Symp on Cavitation. Grenoble, France, 1998.

[21]. H.KATO et Al. Possibility of Quantative Prediction of Cavitation Erosion Without Model Test. International Symposium on Cavitation, Deauville 1995 .

[22]. Y.LECOFFRE, A.ARCHER. A method to Evaluate Cavitation Erosion in Valves. Third Int Symp on Cavitation. Grenoble, France, 1998. 